\title{
Static and dynamic aspects of an air-gap capacitor
}

\author{
Dominicus J IJntema and Harrie A C Tilmans \\ MESA Research Institute, University of Twente, PO Box 217, 7500 AE Enschede (Netherlands)
}

(Recelved September 23, 1991, in revised form March 10, 1992, accepted June 16, 1992)

\begin{abstract}
This paper deals with the theory of an air-gap capacitor used as a micromechanical resonator Both static and dynamic aspects are discussed A single-element approach for the electrostatic excitation and capacitive detection of the vibrational motion of the resonators is described The non-linear character of the electrostatic force is accounted for in the static analysis The behaviour of the air-gap capacitor is modelled as a lumped spring-mass system and its limitations are discussed Also an equivalent electrical one-port network is derived, which can be used in a circuit simulation to account for the mechanical behaviour of the resonator The results obtained from the spring-mass system are compared with the results obtained from a more elaborate numencal analysis of the air-gap capacitor The lumped spring-mass system is adequate for modelling the air-gap capacitor
\end{abstract}

\section{Introduction}

The air-gap capacitor consists of two electrodes separated by a gap The gap can be air (or any other gas) or vacuum The structure forms a (micro)mechanical resonator, which can be used as the sensing element in mechanical sensors [1, 2] In this paper only flexurally vibratıng elements are discussed One electrode is formed by the vibrating element, e g, a beam, and the other electrode by a stationary surface in close proximity to the vibrating element $A$ schematic cross section of the structure is shown in Fig 1

The structure is excited by means of the electrostatic force between the capacitor plates The v1brational motion is detected as a change of the capacitance caused by the fluctuating gap spacing Electrostatic excitation/capacitive detection was first described by Nathanson et al in 1967 [3] and

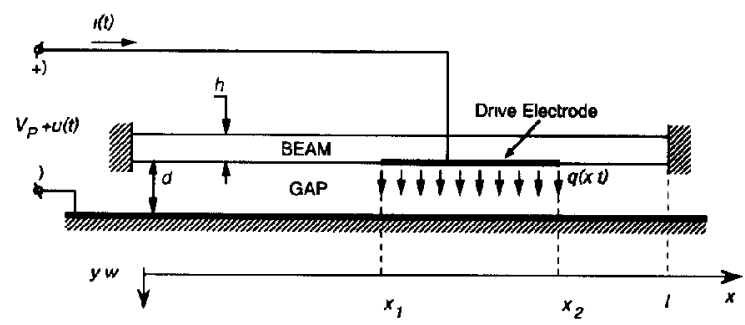

Fig 1 Sketch of an electrostatically excited and capacitively detected beam (air-gap capacitor), driven by means of a single element has been used by several authors since then [4-6] Here, a two-terminal device is described, where a single element is used for both the excitation and detection of the vibration $[5,7,8]$ The structure can be modelled as an electrical one-port network This is in contrast to the two-port approach, where separate elements are used for the excitation and detection of the motion $[4,6]$

In this paper, the effect of the static deflection caused by the $d c$ polarization voltage is included in the analysis of the behaviour of a clampedclamped prismatic beam The pull-in voltage and the resonance frequency of the air-gap capacitor and the modal capacitance of the equivalent electrical network are computed using numerical methods Guidelines are given to derive a lumped spring-mass system for a given air-gap capacitor

\section{Principle of operation}

The air-gap capacitor typically consists of a clamped-clamped beam with a top drive electrode extending from $x=x_{1}$ to $x=x_{2}$, see $F_{1} g 1$ The bottom electrode is formed by a stationary surface The a $\mathrm{c}$ drive voltage $u(t)$ is superumposed onto a $\mathrm{d} c$ polarization voltage $V_{\mathrm{P}}$ to avoid excitation at twice the drive frequency [3] The vibrational motion will change the gap spacing and therefore also the capacitance Because of the 
changing capacitance, an a c current will flow through the air-gap capacitor The a c current is a measure of the amplitude of the vibration The polarization voltage $V_{\mathrm{P}}$ across both electrodes will cause an electrostatic force between the top electrode and the bottom electrode which is inversely proportional to the square of the gap spacing Due to the non-linear behaviour of the electrostatic force, there is a possibility of instability, $1 \mathrm{e}$, collapse of the structure An axial strain applied to the beam will change the resonance frequency, and in this way the alr-gap capacitor can be used as a strain-sensing element [2] Typical dimensions of a micromechanical air-gap resonator are gap spacIng $=1-2 \mu \mathrm{m}$, length $=100-500 \mu \mathrm{m}$, width $=10$ $100 \mu \mathrm{m}$ and thickness $=1-3 \mu \mathrm{m}$

\section{Theoretical model}

The differential equation of motion governing the deflection $W(x, t)$ of a prismatic beam with a rectangular cross section, subjected to an axial tensile load $N$ and a transverse electrostatic drive load $q(x, t)$ can be expressed as

$$
\begin{aligned}
& E^{\prime} I \frac{\partial^{4} W(x, t)}{\partial x^{4}}-N \frac{\partial^{2} W(x, t)}{\partial x^{2}} \\
& \quad+\varrho b h \frac{\partial^{2} W(x, t)}{\partial t^{2}}+c \frac{\partial W(x, t)}{\partial t}=q(x, t)
\end{aligned}
$$

where $E^{\prime}, I, \varrho$ and $c$ are the effective Young's modulus, second moment of inertia, specific mass and the viscous drag parameter, respectively, and $b, h, x$ and $t$ are the width and thickness of the beam, position along the beam length and time, respectively For wide beams $(b>5 h)$ the effective Young's modulus equals $E /\left(1-v^{2}\right)$ [9], where $E$ and $v$ are Young's modulus and Poisson's ratio, respectively The air-gap capacitor is excited by applyıng an a $\mathrm{c}$ drive voltage $u(t)$ superimposed on a $\mathrm{d} c$ polarization voltage $V_{\mathrm{P}}[3-5,7]$ The polarization voltage $V_{\mathrm{P}}$ causes a static deflection $y_{\text {stat }}(x)$ of the beam The overall deflection $W(x, t)$ can be written as a superposition of the static deflection $y_{\text {stat }}(x)$ and the dynamic deflection $w(x, t)$ $W(x, t)=y_{\text {stat }}(x)+w(x, t)$ Assumıng the direction of the electric field vector to be perpendicular to the $x$-axis along the entire beam length (F1g 1) and for $w(x, t) \ll d-y_{\text {stat }}(x)$ and $u(t)^{2} \ll V_{\mathrm{P}}^{2}$, the electrostatic load can be expressed as a superposi- tion of a static load $q_{\mathrm{d} c}(x)$ and a dynamic load $q_{\mathrm{a} \mathrm{c}}(x)$

$$
\begin{aligned}
q(x, t) & =\frac{1}{2} \frac{\varepsilon_{0} \varepsilon_{\mathrm{r}} b\left[V_{\mathrm{P}}+u(t)\right]^{2}}{\left[d-y_{\mathrm{stat}}(x)-w(x, t)\right]^{2}} \\
& \approx \frac{1}{2} \frac{\varepsilon_{0} \varepsilon_{\mathrm{r}} b V_{\mathrm{P}}^{2}}{\left[d-y_{\text {stat }}(x)\right]^{2}}+\frac{\varepsilon_{0} \varepsilon_{\mathrm{r}} b u(t) V_{\mathrm{P}}}{\left[d-y_{\mathrm{stat}}(x)\right]^{2}} \\
& \equiv q_{\mathrm{d} \mathrm{c}}(x)+q_{\mathrm{ac}}(x, t)
\end{aligned}
$$

where $\varepsilon_{0}, \varepsilon_{\mathrm{r}}$ and $d$ are the dielectric constant of vacuum, the relative dielectric constant of the gap medium and the zero-voltage gap spacing, respectively

\section{Statıc behaviour}

The attractive electrostatic force $q_{\mathrm{d}}(x)$ caused by the polarization voltage $V_{\mathrm{P}}$ is inversely proportional to the square of the gap spacing $d-y_{\text {stat }}(x)$ (eqn (2)) An increase of the deflection of the beam results in a decrease of the gap spacing and thus in an increase of the electrostatic force If $V_{\mathrm{P}}$ exceeds the so-called pull-in voltage $V_{\mathrm{PI}}$, the deflection does not reach an equilibrium position and will contınue to increase until physical contact is made with the stationary bottom electrode The system is mechanically unstable [3] For polarization voltages smaller than $V_{\mathrm{PI}}$, the deflection $y_{\text {stat }}(x)$ will reach an equilibrium position and the electrostatic force balances the restoring force caused by the stiffness of the beam The static deflection $y_{\text {stat }}(x)$ can be found by solving the non-lınear differentıal eqn (1), thereby settıng the tıme derivatives equal to zero An analytical closed-form solution of the deflection curve $y_{\text {stat }}(x)$ cannot be found, instead an iterative numerical procedure can be used The iterative solution process can be expressed as

$$
\begin{aligned}
E^{\prime} I \frac{\mathrm{d}^{4} y_{n}(x)}{\mathrm{d} x^{4}}= & \frac{1}{2} \frac{\varepsilon_{0} \varepsilon_{\mathrm{r}} b V_{\mathrm{P}}^{2}}{\left[d-y_{n-1}(x)\right]^{2}} \\
& +N \frac{\mathrm{d}^{2} y_{n-1}(x)}{\mathrm{d} x^{2}} \quad n=2,3,4,
\end{aligned}
$$

$y_{1}(x)=0$

$\frac{\mathrm{d}^{2} y_{1}(x)}{\mathrm{d} x^{2}}=0$

where $y_{n}(x)$ is the $n$th iteration of the static deflection, satisfying the boundary conditions of the beam For the numerical computations, the beam is divided into a finite number of length intervals 
The fourth to the first derivatives and the displacement at every point in the interval are computed using the backward Euler method [10] For polarization voltages $V_{\mathrm{P}}$ smaller than the pull-in voltage $V_{\mathrm{PI}}$, the algorithm of eqn (3) w1ll converge to the equilibrium static deflection of the beam For polarization voltages exceeding the pull-in voltage, the static deflection will contınue to increase and finally equals the gap distance $d$ For small axial loads $N$ the algorithm described by eqn (3) works properly For moderate axial loads, however, only a fraction of the change in $y_{n}$ and $y_{n}{ }^{(2)}$ between two iterations should be used to ensure convergence For large axial loads, the clamped-clamped beam can be modelled as a string, $1 \mathrm{e}$, Ignoring the term with the fourth derivative in eqn (1) The algorithm described in eqn (3) can now be written as

$N \frac{\mathrm{d}^{2} y_{n}(x)}{\mathrm{d} x^{2}}=-\frac{1}{2} \frac{\varepsilon_{0} \varepsilon_{\mathrm{r}} b V_{\mathrm{P}}^{2}}{\left[d-y_{n-1}(x)\right]^{2}} \quad n=2,3,4$,

$y_{1}(x)=0$

The numerical solution methods described above can be used for polarization voltages close to $V_{\mathrm{PI}}$ In normal operation of the air-gap capac1tor applied as a resonator, the polarization voltage will be much smaller than the pull-in voltage, resulting in a deflection of the beam that is small compared to the gap spacing In this case, the load given by eqn (2) can be linearized, and a set of first-order differential equations is obtained, which can be solved numerically with known algorithms [10] Other methods based on minimum-energy principles, where an approximate shape function for the deflection of the beam is assumed, can also be used [11]

\section{Dynamic behaviour}

The steady-state solution of eqn (1), for a beam with length $l$, driven by a harmonic driving load $q_{\mathrm{ac}}(x, t)=q_{\mathrm{ac}}(x) \exp (\mathrm{j} \omega t)$ can be obtained using a modal analysis $[8,12,13]$

$$
\begin{aligned}
& w(x, t) \\
& =\sum_{n=1}^{\infty} \frac{\phi_{n}(x) \int_{x_{1}}^{x_{2}} \psi_{n}(x) q_{\mathrm{a} \mathrm{c}}(x) \mathrm{d} x}{\omega_{n}{ }^{2} M_{n}\left[1+\frac{1}{Q_{n}}\left(\frac{\mathrm{j} \omega}{\omega_{n}}\right)+\left(\frac{\mathrm{j} \omega}{\omega_{n}}\right)^{2}\right]} \exp (\jmath \omega t)
\end{aligned}
$$

where

$M_{n}=\varrho b h \int_{0}^{l} \phi_{n}(x)^{2} \mathrm{~d} x \quad$ (generalized mass)

and $\phi_{n}(x)$ and $\omega_{n}$ are the mode shape function and the natural frequency of the $n$th mode, respectively, which can be found by solving the eigenvalue problem associated with eqn (1) [14], and $Q_{n}$ is the quality factor of the $n$th mode Optrmal excitation of a particular mode is achieved when the integral in the numerator is maximal On the other hand, suppression of a certain mode is achieved by making the integral very small or, better, zero This can be done by a proper choice of the electrode configuration To excite the fundamental mode $(n=1)$, one can take a symmetric electrode configuration with respect to the centre of the beam In this way all the asymmetric modes will be suppressed Theoret1cally, it is possible to excite the fundamental symmetric mode with a lower efficiency compared to the optimal excitation of the fundamental mode, meanwhile suppressing the second mode (asymmetric), the third mode (symmetric), the fourth mode (asymmetric) and all higher-order asymmetric modes This is achieved by designing a symmetric electrode configuration with respect to the centre of the beam in such a way that the integral in the numerator of eqn (5) equals zero for the third mode

To derive an electrical one-port network of the arr-gap capacitor of Fig 1, the admittance seen at the electrical terminals is derived [8] The small signal detection current $t(t)$ flowing into the port is given by (for $u(t) \ll V_{\mathrm{P}}$ )

$$
l(t) \approx C_{0} \frac{\mathrm{d} u(t)}{\mathrm{d} t}+V_{\mathrm{P}} \frac{\mathrm{d} C(t)}{\mathrm{d} t}
$$

where $C_{0}$ is the static capacitance, given by

$$
C_{0}=\varepsilon_{0} \varepsilon_{\mathrm{r}} b \int_{x_{1}}^{x_{2}} \begin{gathered}
\mathrm{d} x \\
d-y_{\text {stat }}(x)
\end{gathered}
$$

and $C(t)$ is the momentary capacitance For vibrational amplitudes that are small compared to the gap spacing, the time derivative of the momentary 
capacitance is given by

$$
\begin{aligned}
\frac{\mathrm{d} C(t)}{\mathrm{d} t} & =\varepsilon_{0} \varepsilon_{\mathrm{r}} b \frac{\mathrm{d}}{\mathrm{d} t} \int_{x_{1}}^{x_{2}} \frac{\mathrm{d} x}{d-y_{\mathrm{stat}}(x)-w(x, t)} \\
& \approx j \omega \varepsilon_{0} \varepsilon_{\mathrm{r}} b \int_{x_{1}}^{x_{2}} \frac{w(x) \mathrm{d} x}{\left[d-y_{\text {stat }}(x)\right]^{2}}
\end{aligned}
$$

where $w(x)$ is the amplitude of the vibration, $w(x, t)=w(x) \exp (\mathrm{j} \omega t)$ The approximation in eqn (8) applies for $w(x) \ll d-y_{\text {stat }}(x)$ Combining eqns (2), (5), (6), (7) and (8) yields an expression for the admittance $Y(j \omega)$ of the alr-gap capacitor

$$
\begin{aligned}
Y(\jmath \omega) \equiv & \frac{l}{u}=\jmath \omega C_{0} \\
& +\sum_{n=1}^{\infty} \jmath \omega \frac{\left(\varepsilon_{0} \varepsilon_{\mathrm{r}} b V_{\mathrm{P}}\right)^{2}}{\omega_{n}{ }^{2} M_{n}\left[1+\frac{1}{Q_{n}}\left(\frac{\jmath \omega}{\omega_{n}}\right)+\left(\frac{\jmath \omega}{\omega_{n}}\right)^{2}\right]} \\
& \times\left[\int_{x_{1}}^{x_{2}} \frac{\phi_{n}(x) \mathrm{d} x}{\left[d-y_{\text {stat }}(x)\right]^{2}}\right]^{2}
\end{aligned}
$$

The expression for the admittance of the air-gap capacitor given by eqn (9) can be represented by the one-port network shown in Fig $2[8,13]$ This equivalent electrical network can subsequently be used in a circuit simulation, e g, SPICE, as a convenient way to account for the mechanical behaviour of the resonator The static capacitance

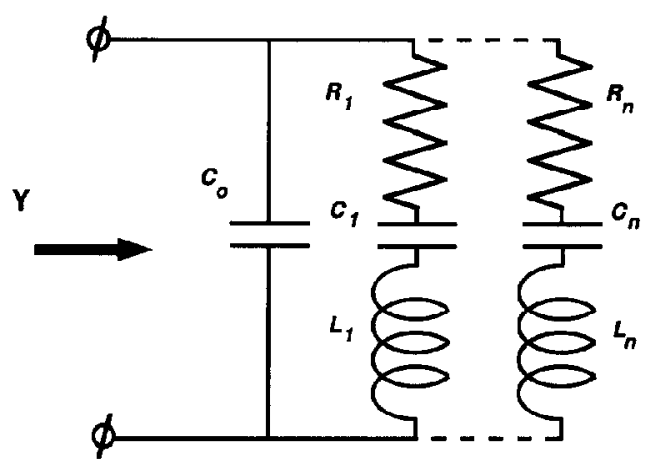

Fig 2 Equivalent electrical one-port network of the arr-gap capac1tor shown in Fig 1
$C_{0}$ is given by eqn (7) The dynamic component values of the electrical network are given by

$C_{n}=\frac{\left(\varepsilon_{0} \varepsilon_{\mathrm{r}} b V_{\mathrm{P}}\right)^{2}}{\omega_{n}{ }^{2} M_{n}}\left[\int_{x_{1}}^{x_{2}} \frac{\phi_{n}(x) \mathrm{d} x}{\left[d-y_{\mathrm{stat}}(x)\right]^{2}}\right]^{2}$

$L_{n}=\frac{1}{\omega_{n}^{2} C_{n}}$

and

$R_{n}=\frac{1}{Q_{n}}\left(\frac{L_{n}}{C_{n}}\right)^{1 / 2}$

Mechanical instability, caused by polarization voltages exceeding $V_{\mathrm{PI}}$, has already been discussed in the previous Section Another effect of the polarization voltage is the lowering of the natural frequency of the air-gap capacitor due to the gradient in the transverse electrostatic force, which varies with the polarization voltage [3] A good approximation for the resonance frequency can be found from an analysis based on Rayleigh's energy method [12] Besides the contribution to the potential energy due to bending and axial deformation of the beam, an additional term $E_{\text {load }}\left(V_{\mathrm{P}}\right)$, representing the change in energy of the electric field in the gap due to the vibration, must be included Using a first-order approximation, this term can be expressed as

$$
\begin{aligned}
E_{\text {load }}\left(V_{\mathrm{P}}\right) \approx & -\left.\int_{x_{1}}^{x_{2}} \int_{0}^{\tilde{w}(x)} \frac{\partial q(x, t)}{\partial w(x, t)}\right|_{w(x, t)=0} \\
& \times w(x) \mathrm{d} w(x) \mathrm{d} x \\
= & -\left.\frac{1}{2} \int_{x_{1}}^{x_{2}} \frac{\partial q(x, t)}{\partial w(x, t)}\right|_{w(x, t)=0} \tilde{w}^{2}(x) \mathrm{d} x \\
\approx & -\frac{1}{2} \int_{x_{1}}^{x_{2}} \frac{\varepsilon_{0} \varepsilon_{\mathrm{r}} b V_{\mathrm{P}}^{2}}{\left[d-y_{\text {stat }}(x)\right]^{3}} \tilde{w}^{2}(x) \mathrm{d} x
\end{aligned}
$$

where $\tilde{w}(x)$ is the assumed shape of the particular mode and $q(x, t)$ is given by eqn (2) The last approximation in eqn (13) applies for $u(t) \ll V_{\mathrm{P}}$ and $w(x) \ll d-y_{\text {stat }}(x)$ The angular resonance frequency $\omega_{n}\left(N, V_{\mathrm{P}}\right)$ of mode $n$ as a function of the axial load $N$ and the polarization voltage $V_{\mathrm{P}}$ can 
be expressed as

$$
\omega_{n}^{2}\left(N, V_{\mathrm{P}}\right)=\frac{E^{\prime} T \int_{0}^{l}\left(\frac{\mathrm{d}^{2} \tilde{w}(x)}{\mathrm{d} x^{2}}\right)^{2} \mathrm{~d} x+N \int_{0}^{l}\left(\frac{\mathrm{d} \tilde{w}(x)}{\mathrm{d} x}\right)^{2} \mathrm{~d} x-\varepsilon_{0} \varepsilon_{\mathrm{r}} b V_{\mathrm{P}}^{2} \int_{x_{1}}^{x_{2}} \frac{\tilde{w}^{2}(x)}{\left[d-y_{\mathrm{stat}}(x)\right]^{3}} \mathrm{~d} x}{\varrho b h \int_{0}^{l} \tilde{w}^{2}(x) \mathrm{d} x}
$$

The first two terms in the numerator represent the potential energy due to bending and axial deformation of the beam, respectively The last term of the numerator represents the change in potential energy due to work done by the electrostatic force This term makes the resonance frequency dependent on the polarization voltage $V_{\mathbf{P}}$ At the pull-in voltage, the fundamental frequency $(n=1)$ equals zero This behaviour is similar to the buckling phenomenon [14] The resonance frequency of the higher-order modes will not equal zero at the pull-in voltage

To indicate the dependence of the angular resonance frequency of the fundamental mode $(n=1)$ $\omega_{1}$ on the applied axial strain $\varepsilon=N /(E b h)$ and on the polarization voltage $V_{P}$, the angular resonance frequency of the fundamental mode can conveniently be written as

$$
\omega_{1}^{2}\left(\varepsilon, V_{\mathbf{P}}\right)=\omega_{1}{ }^{2}(\varepsilon)+\frac{E_{\text {load }}\left(V_{\mathrm{PI}}\right)}{\frac{1}{2} \varrho b h \int_{0}^{1} \tilde{w}^{2}(x) \mathrm{d} x}
$$

where

$\omega_{1}^{2}(\varepsilon)=\omega_{10}^{2}\left[1+0295 \varepsilon\left(1-\nu^{2}\right)\left(\frac{l}{h}\right)^{2}\right]$

and $\omega_{10}$ is the angular resonance frequency of the fundamental mode for zero applied strain The expression above (eqn (16)) is found from an analysis based on Rayleigh's energy method, where the mode shape for zero applied axial load is taken as the approximate shape function [2]

\section{Spring-mass model}

Because of the complexity of the model of the air-gap capacitor, which makes it unsuitable for quick design calculations, it would be attractive to have a simple model to predict the static and dynamic behaviour of the resonator with sufficient accuracy A lumped spring-mass system, see Fig 3, provides such a model [3], but the accuracy of the model has never been verified The lumped spring-mass system is dimensioned in such a way that the static and dynamic behaviours of the spring-mass system resemble the static and dynamic behaviours of the air-gap capacitor in a narrow frequency regime around the fundamental mode Also, the sprng-mass system has to be dimensioned without the necessity for difficult calculations The zero-voltage gap spacing $d$ and the electrode area $A=\left(x_{2}-x_{1}\right) b$ are chosen to be the same for both systems An appropriate choice for the spring constant $K_{0}$ of the spring-mass system would be the static spring constant seen at the centre of the beam for zero applied axial load For a drive electrode with a uniform width, the spring constant $K_{0}$ could be defined as the spring constant of a beam subjected to uniform load $q_{0}$

$K_{0}=\frac{\int_{x_{1}}^{x_{2}} q_{0} \mathrm{~d} x}{\left.y_{\text {stat }}\left(\frac{l}{2}\right)\right|_{q_{0}}}$

For a clamped-clamped beam with the drive electrode extending along the entire beam length, $K_{0}$ is given by $K_{0}=384 E^{\prime} I / l^{3}$ An additional term $K_{\varepsilon}$ given by eqn (20) is added to include the effect of the axial strain The overall spring constant $K$ is given by $K=K_{0}+K_{\varepsilon}$, see also Fig 3

The mass of the spring-mass system is chosen in such a way that its resonance frequency equals the fundamental resonance frequency of the air-gap capacitor with zero applied polanzation voltage, as given by eqn (16) For the lumped spring-mass system the angular resonance frequency $\omega_{1}^{\text {sm }}(\varepsilon)$, with zero applied polarization voltage, is given by

$\omega_{1}^{\mathrm{sm}}(\varepsilon)=\left(\frac{K}{M}\right)^{1 / 2}=\left(\frac{K_{0}}{M}+\frac{K_{\varepsilon}}{M}\right)^{1 / 2}$ 


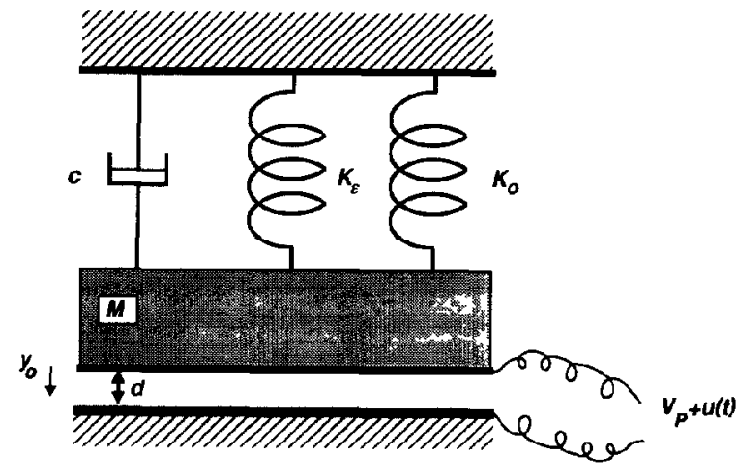

Fig 3 Sketch of the equivalent spring-mass system that is used as a model for the arr-gap capacitor and can be used for quick design calculations $K_{0}$ is the static spring constant seen at the centre of the beam for zero applied axial load An additional spring constant $K_{\varepsilon}$ is added to include the effect of the axial strain

Combining eqns (16) and (18) for $\varepsilon=0$ yields the mass of the spring-mass system

$M=K_{0} / \omega_{10}^{2}$

The spring constant $K_{\varepsilon}$ is found by combining eqns (16), (18) and (19)

$K_{\mathrm{s}}=K_{0} 0295 \varepsilon\left(1-v^{2}\right)\left(\frac{l}{h}\right)^{2}$

The angular resonance frequency of the springmass system $\omega_{1}^{\mathrm{sm}}\left(\varepsilon, V_{\mathrm{P}}\right)$ as a function of the axial strain (applied to the air-gap capacitor) and the polarization voltage can be expressed as [3]

$\omega_{1}^{\mathrm{sm}}\left(\varepsilon, V_{\mathrm{P}}\right)=\omega_{1}^{\mathrm{sm}}(\varepsilon)\left(3-2 \frac{d}{d-y_{0}\left(V_{\mathrm{P}}\right)}\right)^{1 / 2}$

where $y_{0}\left(V_{\mathrm{P}}\right)$ is the static displacement of the spring-mass system caused by the polarization voltage $V_{\mathrm{P}} y_{0}\left(V_{\mathrm{P}}\right)$ can be found by solving the following cubic equation resulting from force equilibrium [3]

$K y_{0}=\frac{1}{2} \frac{\varepsilon_{0} \varepsilon_{\mathrm{r}} A V_{\mathbf{P}}^{2}}{\left[d-y_{0}\right]^{2}} \Rightarrow y_{0}=y_{0}\left(V_{\mathrm{P}}\right)$

The viscous drag parameter $c$ of the spring-mass system can be calculated from the quality factor $Q_{1}$ of the aur-gap capacitor by

$c=\frac{M \omega_{1}^{\mathrm{sm}}\left(\varepsilon, V_{\mathbf{P}}\right)}{Q_{1}}$

An expression for the pull-in voltage $V_{\mathrm{PI}}^{\mathrm{sm}}$ of the spring-mass system can easily be derived by settıng the derivative of the net force acting on the spring- mass system equal to zero [3] Combining this result with the spring constant $K=K_{0}+K_{\varepsilon}$ of a clamped-clamped beam with the drive electrode extending along the entire beam length gives an approximate expression for the pull-in voltage of the beam

$$
\begin{aligned}
V_{\mathrm{PI}}^{\mathrm{sm}} & =\left(\frac{8}{27} \frac{K d^{3}}{\varepsilon_{0} \varepsilon_{\mathrm{r}} A}\right)^{1 / 2} \\
& =107\left\{\frac{E^{\prime} I d^{3}}{c_{0} \varepsilon_{\mathrm{r}} A l^{3}}\left[1+0295 \varepsilon\left(1-v^{2}\right)\left(\frac{l}{h}\right)^{2}\right]\right\}^{1 / 2}
\end{aligned}
$$

The admittance seen at the electrical terminals of the spring-mass system can be derived in a similar way as for the air-gap capacitor The static capacitance $C_{0}^{\text {sm }}$ and modal capacitance $C_{1}^{\mathrm{sm}}$ of the spring-mass system are given by

$C_{0}^{\mathrm{sm}}=\frac{\varepsilon_{0} \varepsilon_{\mathrm{r}} A}{d-y_{0}\left(V_{\mathrm{P}}\right)}$

and

$$
C_{1}^{\mathrm{sm}}=\frac{V_{\mathrm{P}}^{2}\left(C_{0}^{\mathrm{sm}}\right)^{2}}{M \omega_{1}^{\mathrm{sm}}\left(\varepsilon, V_{\mathrm{P}}\right)^{2}\left[d-y_{0}\left(V_{\mathrm{P}}\right)\right]^{2}}
$$

\section{Results}

To have some idea about the accuracy of the results obtained from the spring-mass system, a few examples are presented The equivalent springmass system is dimensioned with the guidelınes given in the last paragraph First the pull-in voltage obtained from the model of the arr-gap capacitor is compared with the pull-in voltage obtained from the spring-mass model

The pull-in voltage $V_{\mathrm{PI}}$ of the alr-gap capacitor is computed with the algonthm described by eqns (3) and (4) The pull-in voltage $V_{\mathrm{PI}}^{\mathrm{sm}}$ of the equivalent spring-mass system is computed from eqn (24) The results of the computation of the pull-in voltages for different beam dimensions are given in Table 1 It turns out that the estımated pull-in voltage $V_{\mathrm{PI}}^{\mathrm{sm}}$ is roughly $10 \%$ lower than the actual pull-in voltage $V_{\mathrm{PI}}$ computed for an air-gap capacitor with the electrode extending along the entire beam length and for small axial strains It can be shown that for higher strain levels and/or an electrode not extending along the enture beam length, the estimation will be more accurate 
TABLE 1 Pull-m voltage and resonance frequency of the aur-gap capacitor and the equivalent spring-mass model for vanous dimensions and axial strains $\left(E=175 \mathrm{GPa}, \varrho=2330 \mathrm{~kg} \mathrm{~m}^{-3}, v=03\right.$ and $\left.\varepsilon_{\mathbf{r}}=1\right)$

\begin{tabular}{llllccc}
\hline $\begin{array}{l}\text { Length } \\
(\mu \mathrm{m})\end{array}$ & $\begin{array}{l}\text { Width } \\
(\mu \mathrm{m})\end{array}$ & $\begin{array}{l}\text { Thickness } \\
(\mu \mathrm{m})\end{array}$ & $\begin{array}{l}\text { Gap } \\
(\mu \mathrm{m})\end{array}$ & Strain & $\begin{array}{l}V_{\mathbf{P I}} \\
(\mathrm{V}) \\
\text { beam }\end{array}$ & $\begin{array}{l}V_{\mathrm{PI}}^{\mathrm{sm}} \\
(\mathrm{V}) \\
\text { lumped }\end{array}$ \\
\hline 500 & 100 & 15 & 10 & 00 & 38 & 34 \\
500 & 100 & 15 & 50 & 00 & 42 & 38 \\
100 & 100 & 15 & 10 & 00 & 94 & 85 \\
500 & 100 & 15 & 10 & $10^{-5}$ & 43 & 39 \\
500 & 100 & 15 & 10 & $10^{-2}$ & 58 & 61 \\
500 & 100 & 60 & 10 & 00 & 30 & 27 \\
\hline
\end{tabular}

The modal capacitance $C_{1}$ and the angular resonance frequency $\omega_{1}\left(\varepsilon, V_{\mathrm{P}}\right)$ of the fundamental mode as a function of the applied axial strain and the polarization voltage are computed for the arrgap capacitor and compared with the modal capacitance $C_{1}^{\mathrm{sm}}$ and the angular resonance frequency $\omega_{1}^{\mathrm{sm}}\left(\varepsilon, V_{\mathbf{P}}\right)$ obtained from the springmass system To calculate the modal capacitance $C_{1}$ and the resonance frequency $\omega_{1}\left(\varepsilon, V_{\mathrm{P}}\right)$, first the static deflection $y_{\text {stat }}(x)$ is computed from eqns (3) or (4) An elghth-order polynomial of the static deflection curve of the beam is generated for a given polarization voltage The polynomial fit of the static deflection is used in eqns (10) and (13) to obtain the modal capacitance $C_{1}$ and $E_{\text {load }}\left(V_{\mathrm{P}}\right)$, respectively For $\phi_{1}(x)$ and $\tilde{w}(x)$, the mode shape of a clamped-clamped beam with an axial strain is used in eqns (10) and (13) [2] For large axial strains $\left(\varepsilon=10^{-2}\right)$, a sine function is used as the approximated mode-shape function The angular resonance frequency is computed numerically from eqn (15) To calculate the modal capacitance $C_{1}^{\mathrm{sm}}$ and the angular resonance frequency $\omega_{1}^{\mathrm{sm}}\left(\varepsilon, V_{\mathrm{P}}\right)$, the static deflection $y_{0}\left(V_{\mathrm{P}}\right)$ of the spring-mass system has to be calculated A closed-form expression of the static deflection can be found by using a first-order Taylor series expansion for the solution of eqn (22), which results in

$y_{0}\left(V_{\mathrm{P}}\right) \approx \frac{1}{2} \frac{\varepsilon_{0} \varepsilon_{\mathrm{r}} A V_{\mathrm{P}}^{2} d}{K d^{3}-\varepsilon_{0} \varepsilon_{\mathrm{r}} A V_{\mathrm{P}}^{2}}$

For polarization voltages up to $08 V_{\mathrm{PI}}^{\mathrm{sm}}$ the error in the static displacement is less than $5 \%$ For a polarization voltage of $09 V_{\mathrm{PI}}^{\mathrm{sm}}$ the error is $10 \%$ In practical situations, where the polarization voltage is much smaller than the pull-in voltage, eqn (27)

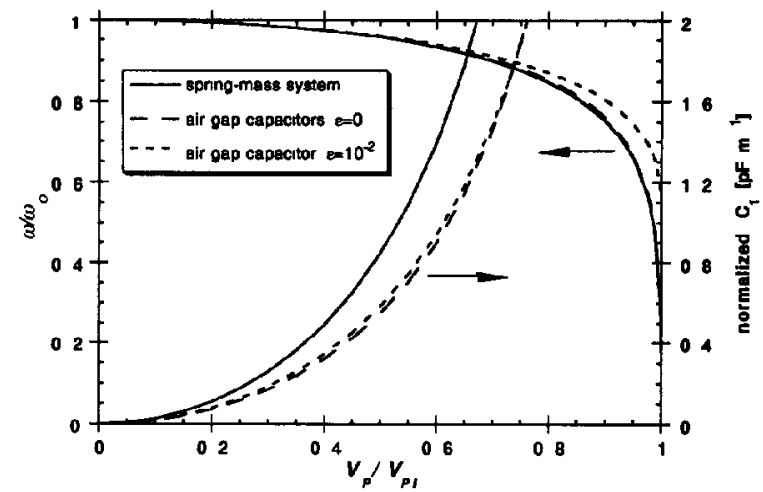

Fig 4 The normalized modal capacitance $\left(C_{1}^{*} d / b l, C_{1}^{s m *} d / A\right)$ and the normalized angular resonance frequency $\left(\omega_{1}\left(\varepsilon, V_{\mathrm{p}}\right) / \omega_{1}(\varepsilon, 0)\right.$, $\left.\omega_{1}^{\mathrm{sm}}\left(\varepsilon, V_{\mathrm{P}}\right) / \omega_{1}^{\mathrm{sm}}(\varepsilon, 0)\right)$ ys the normalized polarization voltage $\left(V_{\mathrm{P}} /\right.$ $\left.V_{\mathrm{PI}}, V_{\mathrm{P}} / V_{\mathrm{PI}}^{\mathrm{sm}}\right)$ of the air-gap capacitor with various dimensions and of the equivalent spring-mass system The curves of the arr-gap capac1tors with zero or small applied axial strains all more or less coincide

will provide a good approximation of the static displacement of the spring-mass system Because the static deflection $y_{0}\left(V_{\mathrm{P}}\right)$ given by eqn (27) is not accurate for polarization voltages close to the pull-in voltage $V_{\mathrm{PI}}^{\mathrm{sm}}$, eqn (22) is solved numerlcally

Figure 4 shows the normalized modal capacitance of the alr-gap capacitor $\left(C_{1}^{*} d / b l\right)$ and the normalized angular resonance frequency of the air-gap capacitor $\left(\omega_{1}\left(\varepsilon, V_{\mathrm{P}}\right) / \omega_{1}(\varepsilon, 0)\right)$ as a function of the normalized polarization voltage for beams of various dimensions and different applied axial strains (see also Table 1) The angular resonance frequency is normalized to the angular resonance frequency with zero applied polarization voltage The curves of the beams with zero and small applied strains all coincide Figure 4 also shows the normalized modal capacitance $\left(C_{1}^{\mathrm{sm} *} d / A\right)$ and the normalized angular resonance frequency $\left(\omega_{1}^{\mathrm{sm}}\left(\varepsilon, V_{\mathrm{P}}\right) / \omega_{1}^{\mathrm{sm}}(\varepsilon, 0)\right)$ calculated from the equivalent spring-mass model The modal capacitance $C_{1}^{\mathrm{sm}}$ found with the help of the spring-mass system 1s roughly a factor 15 larger than the modal capacitor $C_{1}$ of the alr-gap capacitor The resonance frequencies as a function of the polarization voltage of the air-gap capacitor and the springmass system are approximately equal The modal capacitance $C_{1}^{\mathrm{sm}}$ found from the spring-mass model can be used, after correction with a factor 15 , in the electrical equivalent circuit of the oneport resonator 


\section{Conclusions}

A model of the electrostatically driven and capacitively detected resonator based on a singleelement approach is presented, thereby including in the static behaviour the non-linear effects of the electrostatic force Guidelınes are given to dimension a lumped spring-mass system for a given air-gap capacitor, without the need for tedious calculations For engineering purposes, the electrostatically driven resonator can be described sufficiently accurately by a lumped spring-mass system The pull-1n voltage computed from the spring-mass system is roughly $10 \%$ lower than the value computed for the arr-gap capacitor The dependence of the resonance frequency on the normalized polarization voltage is approximately the same for both the spring-mass system and for the air-gap capacitor The equivalent electrical network can be used in circuit simulations to account for the resonator behaviour in a narrow frequency range around the fundamental frequency The electrical network can be dimensioned rapidly with the help of this lumped spring-mass system

\section{Acknowledgement}

The authors would like to thank Professor Jan Fluitman for helpful discussions and suggestions

\section{References}

1 R T Howe, Resonant microsensors, Proc 4th Int Conf SolldState Sensors and Actuators (Transducers '87), Tokyo, Japan, June 2-5, 1987, pp 843-848

2 H A C Tilmans, M Elwenspoek and J H J Fluttman, Micro resonant force gauges, Sensors and Actuators A, 30 (1992) 35-53

3 H C Nathanson, W E Newel, R A Wickstrom and J R Davis, Jr, The resonant gate transistor, IEEE Trans Electron Devices, ED-14 (1967) 117-133

4 R T Howe and R S Muller, Resonant-microbridge vapor sensor, IEEE Trans Electron Devices, ED-33 (1986) 499-506

5 M W Putty, S C Chang, R T Howe, A L Robinson and K D Wise, One-port active polysilicon resonant microstructures, Proe IEEE MEMS'89 Workshop, Salt Lake City, UT, USA, 1989, pp 60-65

6 C Linder, E Zımmerman and N F de Rooy, Capacitıve polysilicon resonator with MOS detection circuit, Sensors and Acruators A, 25-27 (1991) 591-595

7 M W Putty, Polysilicon resonant microstructures, Master's Thests, University of Michigan, 1988

8 H A C Tilmans, D J IJntema and J H J Fluitman, Single element excitation and detection of (micro-) mechanical resonators, Proc 6th Int Conf Soldd-State Sensors and Actuators (Transducers '91), San Francisco, USA, June 24-27, 1991, pp 533-537

9 S P Timoshenko and S Woinowskl-Kre1ger, Theory of Plates and Shells, McGraw-Hill, New York, 2nd edn, 1970, p 5
10 W H Press, B P Flannery, S A Teukolsky and W T Vetterling. Numerical Recipes, The Art of Scientific Computing, Cambridge Unıversity Press, Cambridge, 1987

11 H A C Tilmans and R Legtenberg, Electrostatıcally driven vacuum encapsulated polysilicon resonators Part II Performance, submitted to Eurosensors VI, San Sebastian, Spain, Sept 30-Oct 2, 1992

12 L Meırovitch, Elements of Vibration Analysis, McGraw-Hıll, New York, 1975, Ch 5, pp 190-231

13 H A C Tilmans, Single element excitation and detection of (micro-)mechanical resonators-theory and experiments, to be published

14 S Bouwstra and B Geljselaers, On the resonance frequencies of microbridges, Proc 6th Int Conf Solld-State Sensors and Actuators (Transducers '91), San Franctsco, USA, June 24-27, 1991, pp $538-542$

\section{Biographies}

Dominicus J IJntema was born in 1966 in Joure, The Netherlands $\mathrm{He}$ received a MS degree in electrical engineering from the University of Twente in March 1991 His master's project dealt with a 'differential resonator design using electrostatically excited resonant gauges' In May 1991 he became a research engineer at the MESA Research Institute of the University of Twente, The Netherlands, where he was working on a micromachıned eyepressure regulator for glaucoma patients Currently he is working at Philips National Research Laboratory in Eindhoven

Harrle A C Tilmans was born in 1957 in Elsloo, The Netherlands He received his M S degree in electrical engineering from the University of Twente, Enschede, The Netherlands, in May 1984 In June 1984 he became a temporary research associate at the University of Twente, where he worked on the development of a resonating force sensor In April 1985 he joined the faculty of Electrical and Computer Engineening of Boston University, Boston, USA, as a visiting instructor In August 1986 he became a research assistant at the Wisconsin Center for Applied Microelectronics at the University of Wisconsin, Madison, WI, USA, where he worked on the development of a CMOS process and on the development of polysilicon micromechanical resonators In August 1988 he returned to the The Netherlands, where he joined the MESA Research Institute of the University of Twente as a research associate Currently, he is preparing for his Ph D thesis, on the subject of micromechanical sensors using encapsulated resonant strain gauges, which will be due in January 1993 